\title{
Design and Experiment of a Deformable Bird-inspired UAV Perching Mechanism
}

\author{
Long Bai ${ }^{1} \cdot$ Hao Wang ${ }^{1} \cdot$ Xiaohong Chen $^{1} \cdot$ Jia Zheng $^{2} \cdot$ Liming Xin $^{3} \cdot$ Yupeng Deng $^{1} \cdot$ Yuanxi Sun $^{1}(\mathbb{D}$
}

Received: 19 April 2021 / Revised: 27 August 2021 / Accepted: 27 September 2021 / Published online: 11 November 2021

(c) The Author(s) 2021

\begin{abstract}
Energy consumption and acoustic noise can be significantly reduced through perching in the sustained flights of small Unmanned Aerial Vehicles (UAVs). However, the existing flying perching robots lack good adaptability or loading capacity in unstructured environments. Aiming at solving these problems, a deformable UAV perching mechanism with strong adaptability and high loading capacity, which is inspired by the structure and movements of birds' feet, is presented in this paper. Three elastic toes, an inverted crank slider mechanism used to realize the opening and closing movements, and a gear mechanism used to deform between two configurations are included in this mechanism. With experiments on its performance towards different objects, Results show that it can perch on various objects reliably, and its payload is more than 15 times its weight. By integrating it with a quadcopter, it can perch on different types of targets in outdoor environments, such as tree branches, cables, eaves, and spherical lamps. In addition, the energy consumption of the UAV perching system when perching on objects can be reduced to 0.015 times that of hovering.
\end{abstract}

Keywords Perching mechanism $\cdot$ Bionic design $\cdot$ UAV $\cdot$ Flying robot $\cdot$ Grasper

\section{Introduction}

With the development of flight control algorithms, sensors, microprocessors, MEMS, and other related technologies, the Unmanned Aerial Vehicle (UAV) technology has developed rapidly. Because of its excellent properties, such as simple deployment, low maintenance cost, high maneuverability, and hovering capability, UAV has been widely used in intelligence surveillance and reconnaissance, traffic monitoring and management, search and rescue, network and communication, infrastructure inspections and many other fields [1-3]. It has played a significant role in those fields. However, due to its size and loading limitations, there are limited onboard power supplies in the UAV, which usually results in

Yuanxi Sun

sunyuanxi@cqu.edu.cn

1 State Key Laboratory of Mechanical Transmission, Chongqing University, Chongqing 400030, China

2 College of Advanced Manufacturing Engineering, Chongqing University of Posts and Telecommunications, Chongqing 400065, China

3 School of Computer Engineering and Science, Shanghai University, Shanghai 200444, China a short flight time. Meanwhile, great acoustic noise would be caused by the interaction between propellers and air during the flight, which is not suitable for covert tasks like surveillance and reconnaissance. In fact, if the UAV can fly and perch like birds with its rotors being partially or completely shut down in those tasks, then its endurance time can be greatly improved and the noise can also be reduced. Thus, the flying perching mechanism is a practical way to solve the existing problems of UAVs.

There are many researches on the flying perching mechanism worldwide. At present, the perching methods can be divided into two types: non-mechanical and mechanical types. The non-mechanical type refers to that the perching or attaching force is mainly produced by non-mechanical methods, such as the air pressure difference [4-6], dry adhesive [7-9], magnetic induction [10, 11], and electrostatic induction [12]. For example, Liu et al., [4] presented a batlike robot that can adhere to various vertical wall surfaces or ceilings with low power consumption by using a vacuum rotor with a centrifugal pump and a sealed chamber producing aerodynamic attraction. Kalantari et al., [7] presented a method that enables quadrotor Micro Air Vehicle (MAV) to autonomously perch on and take off from smooth vertical surfaces with a dry adhesive gripper, which has shown the 
advantages of simple control and high success rate. Roberts et al., [10] presented a way of relieving the energy problem in aerial exploration within indoor environments by a ceiling attachment which mainly depends on a small toroidal magnet. Graule et al., [12] proposed a controllable attaching principle to achieve small-scale MAV perching by electrostatic adhesion. The insect-type robot they designed can repeatedly perch and detach nearly on any material while only requires approximately three orders of magnitude less power than that required in sustained flight. Although those non-mechanical perching methods can achieve successful perching in specific scenarios, they generally have high requirements on the surfaces of the perched objects, which has limited their applications. Electrostatic adhesion, as a novel perching method, is advantageous in its strong adaptability and small mechanical fatigues. However, it is only suitable for micro-flying robots because its adhesive force is very small.

In comparison, the mechanical type has better adaptability in outdoor environments. For example, the multimodal robot with two spiny feet designed by Pope et al., [13] can achieve perching on unprepared surfaces such as concrete and stucco walls. The modularized and actuated landing gear framework proposed by Hang et al., [14] can help UAV perch and rest on a wide range of different structures, like street lights and edges or corners of buildings. In addition, various bionic perching mechanisms have been designed by researchers, which are inspired by the birds being able to fly and perch in the wild. Doyle et al., [15], inspired by the adaptation that enables songbirds to sleep on trees without active muscle control, designed a perching mechanism to expand the number of surfaces upon which a rotorcraft can land. By imitating the structure of bird's feet, the mechanism they designed can convert the weight of rotorcraft into tendon tension to passively perch on structures like tree branches without energy consumption. Xie et al., [16] presented a novel bio-inspired cable-driven leg and foot mechanism to extend the capabilities of the UAV. The designed mechanism can help the host UAV perform tasks like landing on, perching on, and taking off from either a flat surface or a non-flat object. They have conducted in-depth research and analysis on the bird's leg and foot structure to support their design. Nadan et al., [17] presented the design, modeling, simulation and testing of a cable-driven landing gear system to help the UAV perform tasks in urban or rural settings that do not have appropriate or sufficiently large space for takeoff and landing. With the folding of a four-bar linkage when the UAV is landing, two opposed, multi-segmented and flexible feet can curl over and wrap around a range of objects, like trees. Although those existing bionic perching mechanisms can make UAV perch on targets like tree branches, they cannot be deformed and adapted to a wider range of objects, such as cables and spherical lamps. Moreover, most of them are installed at the bottom of the aircraft, which will block part of the camera's view and little research has been carried out on the payload capacity and power consumption of the mechanism, which has been deeply studied in this paper.

The main contribution of this paper is that a deformable bird-inspired mechanism for UAV perching is designed to solve the aforementioned problems. It includes an inverted crank slider mechanism used to realize the opening and closing movements, a gear mechanism used to realize the deformation and three elastic toes with excellent passive deformability. Through experiments, it has been proved to be strong in adaptability and high in loading capacity. By integrating it with a quadcopter, the perching mechanism can perch on different types of targets in outdoor environments, such as tree branches, cables, eaves, and spherical lamps. When perching on them, the system's energy consumption can reduce to 0.015 times that of hovering.

The paper is organized as follows. The bionic principles of the perching mechanism are proposed as being inspired by the structure and movements of birds' feet in Sect. 2. The perching mechanism is designed and prepared in Sect. 3. The experiments and analyses about the loading capacity and adaptability of the perching mechanism are carried out in Sect. 4. In Sect. 5, the UAV perching system is integrated and adopted in the dynamic flying perching experiments. Afterward, its power consumption is analyzed and the feasibility is verified. Finally, the work is summarized and future research is discussed in Sect. 6.

\section{Analysis of Bionic Principle}

In nature, birds often perch on branches, eaves, cables, and other elevated structures. On the one hand, they can rest in a comparatively safe and covert environment to save energy; on the other hand, they can continuously discover and observe preys who are entering their surveillance area and then capture food quickly once there is an opportunity. Birds can greatly save energy with perching so that they can carry out long-term preying tasks with high efficiency, which provides a good reference to the energy-saving and noise-reducing measures of flying robots.

\subsection{Design of Toes}

It can be seen from birds' habitat that grasping is a reliable way for UAVs to perch in complex outdoor environments. Typical bird feet (Fig. 1a) are mainly composed of toe bones, toe pads, and claws $[18,19]$. Toe bones are composed of multiple phalanges in series, driven by tendons. Thus, common bionic perching mechanisms adopt multiple joints in series which are driven by cables [15-17]. However, the cable-driven mechanism requires complex joint components 


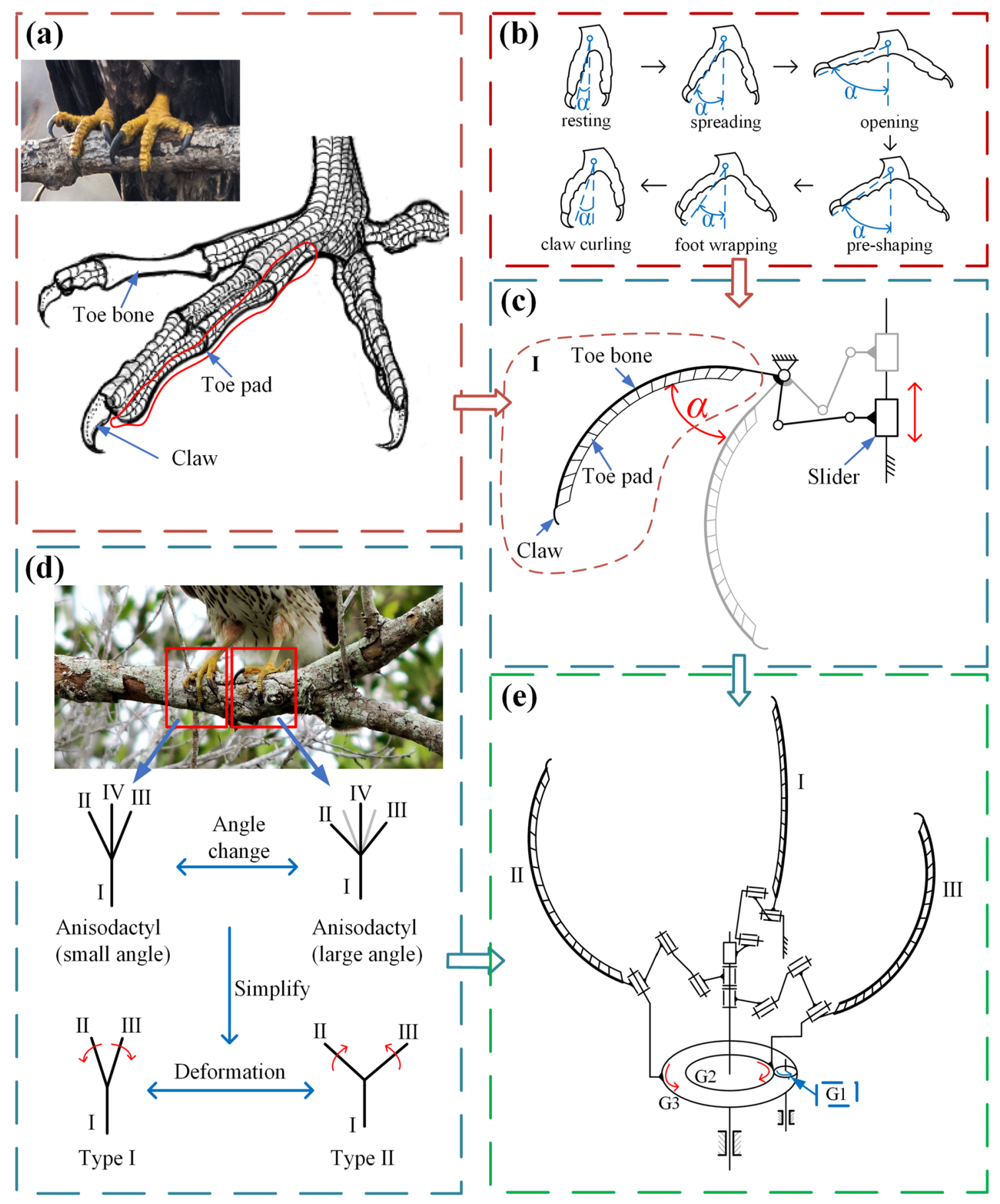

Fig. 1 The bionic principle of the perching mechanism. a The basic structure of bird's toes. b The opening and closing process of bird's foot [18]. c The design concept of toe and toe-rotation mechanism: an inverted crank slider mechanism. d Anisodactyl toe arrangement and principle of configuration-change mechanism: with toe I fixed on

the frame, toe II and toe III rotate in the opposite direction to realize the deformation between type I and type II. e Overall concept of the perching mechanism, with a gear mechanism used to perform the deformation placed in the lower part

and pulleys to guide the movement of cables, which usually leads to the large size and weight of the mechanism. In addition, cables are easily being worn, which usually results in a short lifetime. To avoid those problems and simplify the

structure, an integrated arc structure (left part in Fig. 1c) is proposed. It can be made of elastic materials to realize passive deformation and be adapted to various objects with different shapes and surfaces. 
Toe pads, attaching to the inner side of bones, can not only increase the surface area and roughness to enhance friction force [18] but also perform as a buffer to absorb energy and reduce impact while touching objects. Meanwhile, they are designed as porous to reduce weight and improve adaptability. Claws are sharp curved horny humps on the end of toes. They can generate additional grasping force and reduce relative sliding between toes and perched objects by hooking on or embedding into protrusions or soft parts of the contacting surfaces $[19,20]$. Moreover, they can also help the mechanism perch on targets like cables.

\subsection{Toe-rotation Mechanism}

To realize the opening and closing movements of the foot, the toes need to be rotated at a certain angle (Fig. 1b). There is a compact structure in the inverted crank slider mechanism, which can convert the reciprocating linear motion of sliders into the rotary motion of the output shaft [21], leading to the swing of the output shaft within a certain angle. Compared to a tendon-driven system, the inverted crank slider mechanism can provide a higher contact force with much smaller wear and tear [22]. Therefore, it has been widely used in lots of grippers [22-24]. As the basic principle shown in Fig. 1c, the toes can swing within a certain angle by moving the slider up and down.

\subsection{Configuration-change Mechanism}

The toe arrangement of birds can be divided into several main types [19], which are anisodactyl, zygodactyl, heterodactyl, syndactyly, and pamprodactyl types. The anisodactyl toe arrangement is the most common one, with three toes pointing forward and one toe pointing backward. This type of toe arrangement can be well adapted to grasping tree branches and aiding birds in landing or perching on different structures [16]. Therefore, the anisodactyl toe arrangement is selected as the main configuration of our perching mechanism. Instead of three toes pointing forward, only two toes are pointing forward in our design as it can reduce the complexity and overall weight of the perching mechanism without losing the effective grasping ability.

By observing the angles between toes when birds are perching, it can be found that the angle is not fixed but can be adjusted within a certain range. According to the shapes of common objects, such as cylinders, spheres and cubes, the mechanism's configuration is designed as two types, type I and type II (Fig. 1d). In type I, three toes are divided into two groups which are distributed oppositely. This type is mainly used to perch on cylindrical objects. In type II, three toes are evenly distributed, which are mainly for perching on spherical objects. When performing the deformation between type I and type II, toe I is fixed on the base, toe II and toe III rotate in the opposite direction. To reduce the number of motors, a gear mechanism (lower part in Fig. 1e) is used to drive the two toes simultaneously which are rotated oppositely. In this mechanism, gear G1 is mounted on the motor. When it rotates, the outer gear G3 fixed with toe II rotates in the same direction, while the inner gear G2 fixed with toe III rotates in the opposite direction. In this way, the configuration can be changed between type I and type II.

\section{Structural Design}

The designed perching mechanism (Fig. 2a) comprises four parts: base, configuration-change mechanism, toe-rotation mechanism, and toes. The base is used for installing the Direct Current (DC) motor, which is used to drive the gear of the configuration-change mechanism, and the linear motor to drive the slider of the toe-rotation mechanism. The toe is composed of toe bone, toe pad, and claw. Among them, the toe bone is made of quenched $65 \mathrm{Mn}$ spring steel, with which the passive deformability of the structure can be greatly improved. The toe pad is cast as porous by the silica gel and curing agent pro ratio in a designed mold. The specific preparation process is shown in Fig. 2aI. With the elasticity and flexibility of silica gel and the porous structure, its adaptability, energy absorption and buffer function can be greatly improved. In addition, the claw is composed of a base printed by a $3 \mathrm{D}$ printer and multiple spines processed and polished from aluminum alloy. Finally, the claw, toe bone, and toe pad are connected by strong adhesive.

The configuration-change mechanism is mainly composed of four parts (Fig. 2aII). When the gear G1 is driven to rotate, the configuration can be alternated between type I and type III (Fig. 2c). The structure of the toe-rotation mechanism is shown in Fig. 2aIII. With the slider's reciprocating linear motion, the toe can be rotated at a certain angle to realize the opening and closing movements of the mechanism (Fig. 2b).

The perching mechanism can be mainly installed in three locations of the aircraft: the top, the bottom, and the side. When it's installed on the side [4, 7], the mechanism needs to be extended a long distance along the fuselage to avoid interference between the mechanism and propellers. Thus, a large deviation will be caused in the barycenter of the robot, which will increase the complexity of control. When it's installed at the bottom of the aircraft [17], the camera's view will be partially blocked, and the lateral stability when perching will be influenced. Regarding these disadvantages, the perching mechanism designed in this paper will be installed on the top of the aircraft, which will enlarge the camera's view and facilitate aircraft in perching in open areas under tree branches in typical natural environments. 
Fig. 2 Structural design. a Structure of the perching mechanism, I: preparation process of the toe pad, II: configuration-change mechanism, III: toe-rotation mechanism. b Open and closed states of the perching mechanism. c Configuration of type I and type II. d Self-locking mechanism

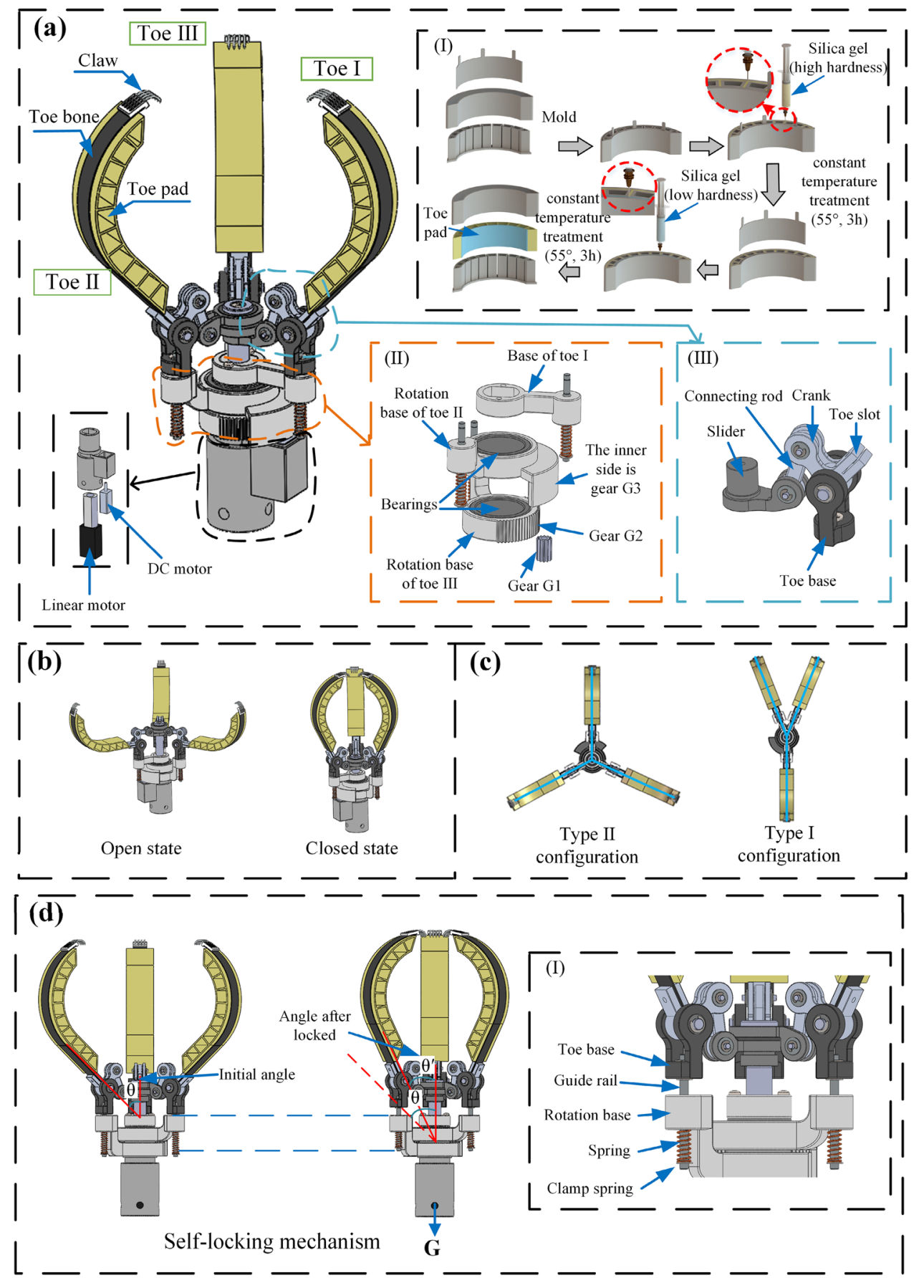

Meanwhile, a self-locking mechanism is designed to save energy and improve reliability, as shown in Fig. 2d. When the perching mechanism is grasping targets, the rotors of the aircraft will stop rotating so that the overall weight will act on the perching mechanism. Then, the slider of the toerotation mechanism will be pulled down so that the perched targets will be grasped tighter. Once the targets are successfully grasped, the perching mechanism will not release with the action of the aircraft's weight even it's powered off, unless it is commanded to do it. It is an important fail-safe and energy-saving mechanism for the system.

To make the perching mechanism adapt to different shapes and sizes of perching targets to the greatest extent, the opening size of the perching mechanism should be as large as possible. In a limited size range, the size of the inverted crank slider mechanism is our main design parameter. The schematic diagram of this mechanism is shown in Fig. 3. 
Fig. 3 Diagram of the inverted crank slider mechanism. a Some important parameters are displayed in this diagram. b The states of two extreme positions
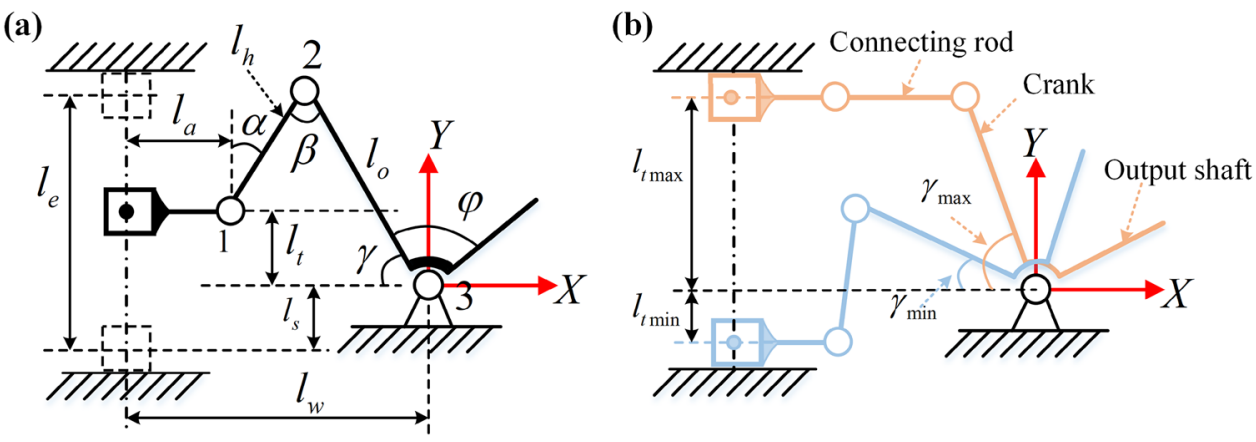

When the slider is at the top, the angle between the crank and the $\mathrm{x}$-axis is the largest, which is $\gamma_{\max }$. From the law of cosines, we have:

$\gamma_{\max }=\arccos \frac{l_{h}^{2}+l_{t}^{2}+2 l_{h} l_{t} \cos \alpha-l_{o}^{2}-\left(l_{w}-l_{a}\right)^{2}}{-2 l_{o}\left(l_{w}-l_{a}\right)}$

Among them, $l_{a}$ is the distance between the slider and the joint $1, l_{h}$ is the length of the connecting rod, $l_{o}$ is the length of the crank, $l_{t}$ is the distance between joint 1 and joint 3 along the y-axis, $l_{w}$ is the distance between the vertical axis of the slider and the joint 3 along the $x$-axis, $\alpha$ is the angle between the connecting rod and the y-axis, it can be obtained by the following formula:

$\alpha=\arccos \frac{l_{o}^{2}-\left(l_{w}-l_{a}\right)^{2}-l_{t}^{2}-l_{h}^{2}}{2 \sqrt{\left(l_{w}-l_{a}\right)^{2}+l_{t}^{2}} \cdot l_{h}}-\arctan \frac{l_{w}-l_{a}}{l_{t}}$

When the slider is at the bottom, the angle is the smallest, which is $\gamma_{\min }$ :

$\gamma_{\min }=\arccos \frac{\left(l_{h}-l_{t} / \cos \alpha\right)^{2}-l_{o}^{2}-\left(l_{w}-l_{a}-l_{t} \tan \alpha\right)^{2}}{-2 l_{o}\left(l_{w}-l_{a}-l_{t} \tan \alpha\right)}$

$\alpha=\arccos \frac{l_{o}^{2}-\left(l_{w}-l_{a}\right)^{2}-l_{t}^{2}-l_{h}^{2}}{2 \sqrt{\left(l_{w}-l_{a}\right)^{2}+l_{t}^{2}} \cdot l_{h}}-\arctan \frac{l_{t}}{l_{w}-l_{a}}$

So, the effective output angle can be expressed as:

$\gamma_{e}=\gamma_{\max }-\gamma_{\min }$

Our goal is to maximize the $\gamma_{\mathrm{e}}$. Considering the overall size and the selection of some parts, we set $l_{a}=15 \mathrm{~mm}$, $l_{w}=30 \mathrm{~mm}, l_{s}=5 \mathrm{~mm}, l_{s}$ is the distance between the lowest position of the slider and the joint 3 along the $y$-axis. The effective stroke of the linear motor is $30 \mathrm{~mm}$, so we have $l_{e}=30 \mathrm{~mm}$. Besides, the angle of $\alpha$ and $\gamma$ should satisfy $\alpha \in[0, \pi / 2], \gamma \in(0, \pi)$. Meanwhile, to make the structure more compact and avoid the overall size from being too large, we set $l_{o} \in(0,30), l_{h} \in(0,20)$. To avoid the existence of dead spots and motion interference, the following relationships need to be satisfied:

$\left(l_{w}-l_{h}-l_{a}\right)^{2}+l_{t \max }^{2}<l_{o}^{2}$

$\left(l_{o}-l_{h}\right)^{2}>\left(l_{w}-l_{a}\right)^{2}+l_{t \max }^{2}$

$l_{o}^{2}-\left(l_{h}-l_{t \min }\right)^{2}<\left(l_{w}-l_{a}\right)^{2}$

After considering all those constraints, we can calculate that $l_{o}=15.12 \mathrm{~mm}, l_{h}=17.08 \mathrm{~mm}$, and the maximum $\gamma_{\mathrm{e}}$ is $89.8^{\circ}$. Besides, the angle $\varphi$ is the fixed angle between the output shaft and the crank. It should satisfy that the perching

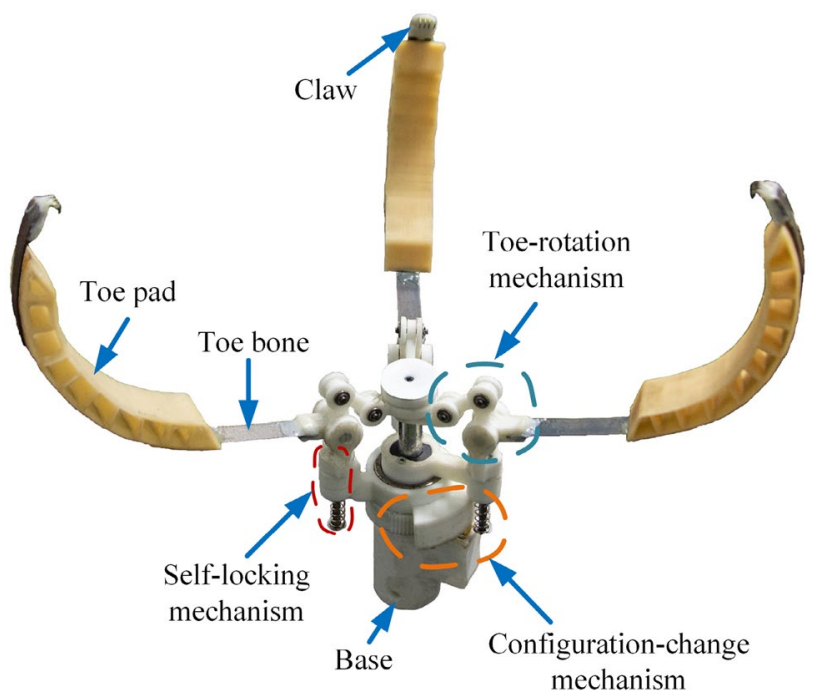

Fig. 4 The prototype of the perching mechanism. It includes three elastic toes composed of toe pads, toe bones and claws, a toe-rotation mechanism that mainly depends on an inverted crank slider mechanism, a configuration-change mechanism that depends on a gear mechanism, a self-locking mechanism, and a base 
Fig. 5 Test platform and control strategy of the perching mechanism. a The perching mechanism grabs the test objects. Then, it is loaded by a motor through a rope, and the loading value is measured by a tension sensor and recorded by a monitor. $\mathbf{b}$ The PID controller is used to control the loading value

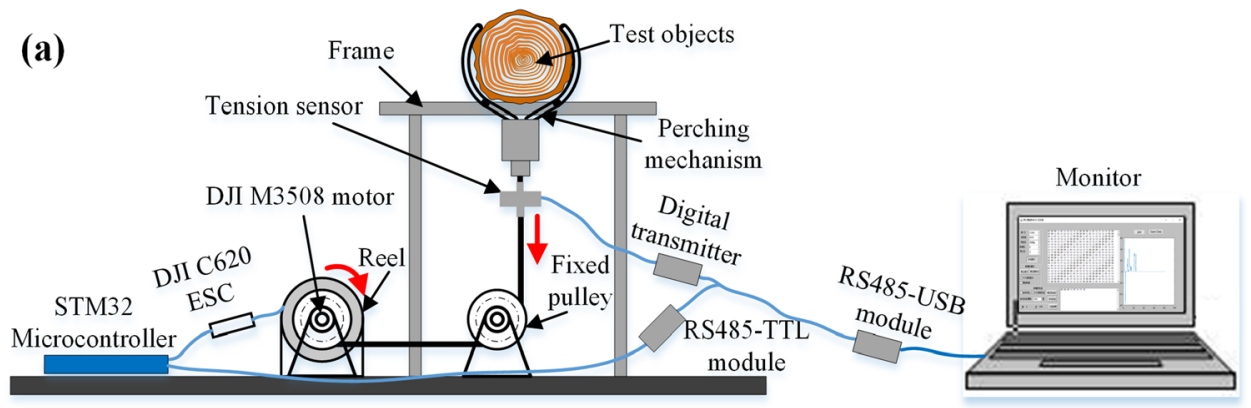

(b)

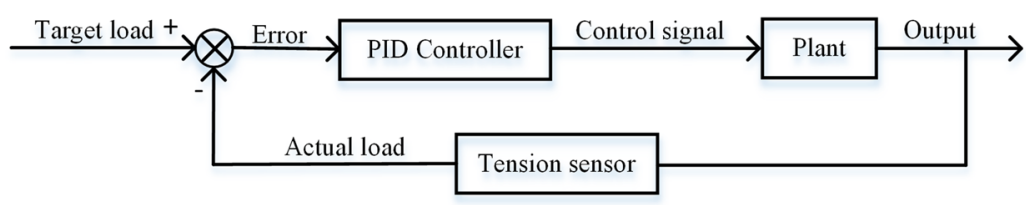

mechanism can be completely closed when the crank is at the smallest angle and be fully opened when the crank is at the largest angle, it is set to $80^{\circ}$ to satisfy these requirements. The toe bones are designed to be composed of a circular arc with a central angle of $110^{\circ}$ and a radius of $45 \mathrm{~mm}$, and a straight line with a length of $40 \mathrm{~mm}$. After testing, this size can be well adapted to different perching targets and can produce sufficient covering force.

The prototype of the perching mechanism is shown in Fig. 4, with $300 \mathrm{~mm}$ of maximum opening diameter after fully opened, $210 \mathrm{~mm}$ of height and $256 \mathrm{~g}$ of weight, respectively.
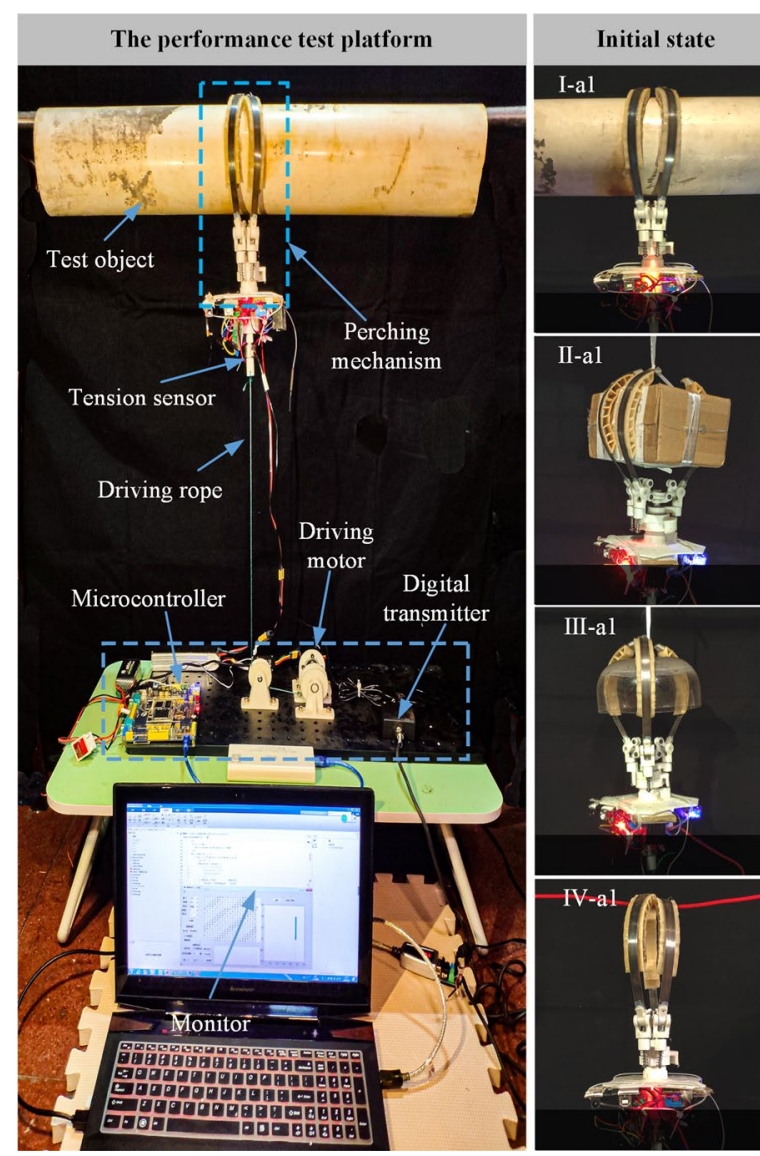

Fig. 6 The performance tests of the perching mechanism. The initial state refers to the load applied on the mechanism is $0 \mathrm{~kg}$, the loaded state refers to the status where the load reaches $4 \mathrm{~kg}$. I: cylindrical

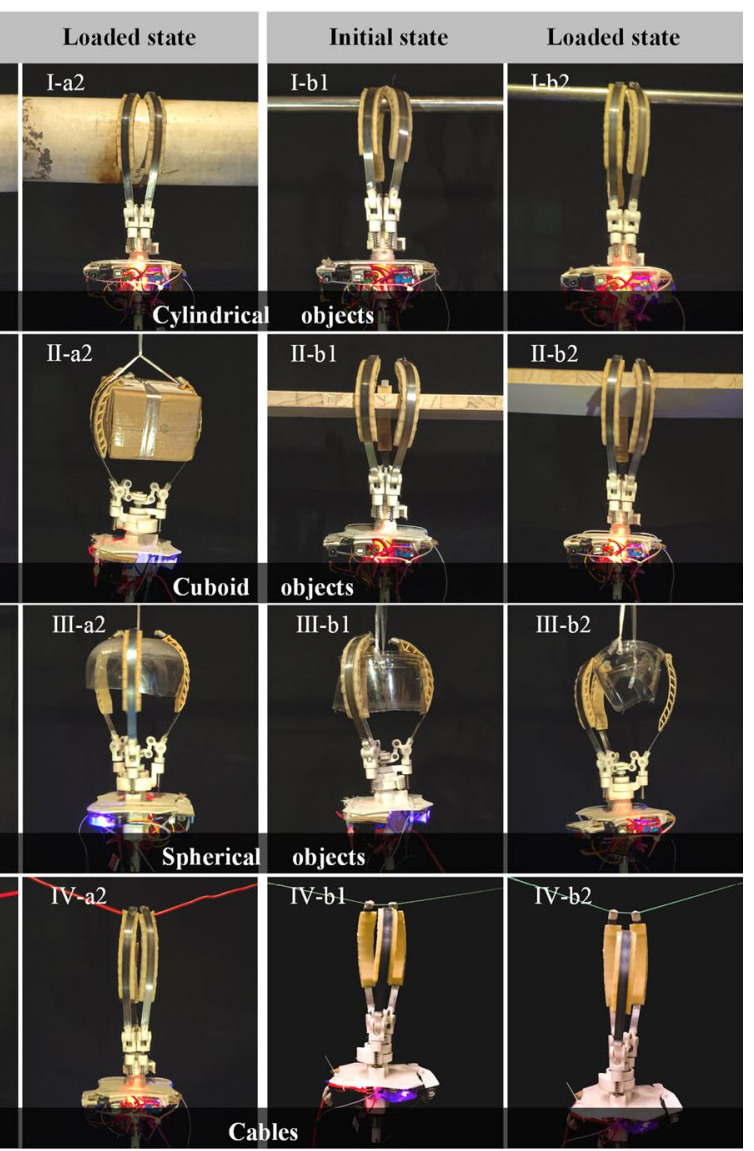

objects (the diameter of I a1/a2 is larger), II cuboid objects (the II a1/ a2 is thicker), III spherical objects (the III a1/a2 is harder), IV cables (the elasticity of IV a1/a2 is better) 
Fig. 7 Loading error zone and loading time of different types of test targets. a Cylindrical objects. The loading curve of the thicker one is smoother, and its loading time is shorter. b Cuboid objects. They have similar trends, but the thicker one has a narrower loading error zone. c Spherical objects. The harder one costs a shorter time. d Cables. The more elastic one cost the longest time among all of the test objects, and its loading error zone is much broader

Fig. 8 Prototype and hardware structure of the system. a The prototype of the integrated flight-perching robot. b The system's hardware structure. It's mainly composed of two parts, the flight system and the perching system
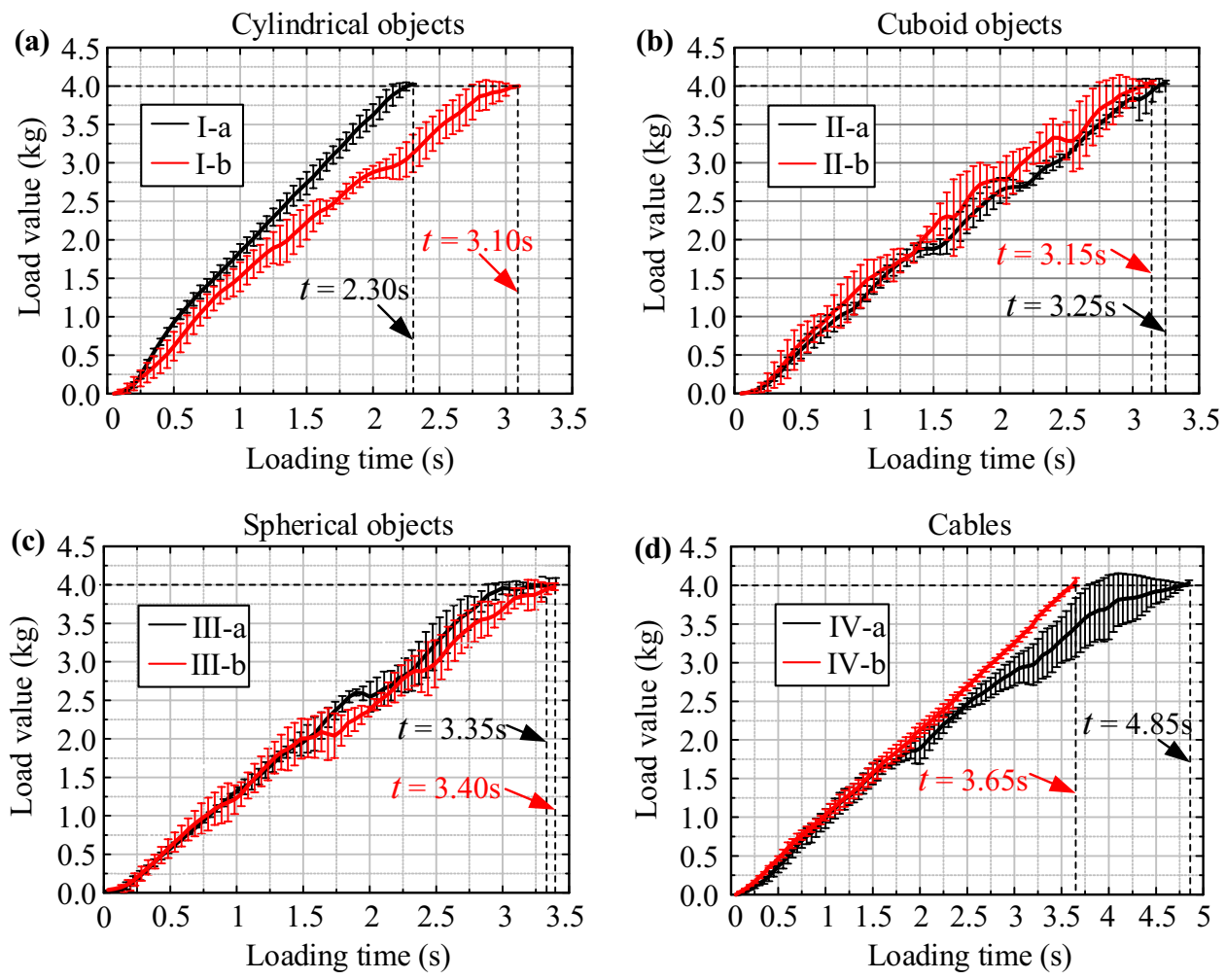

(a)

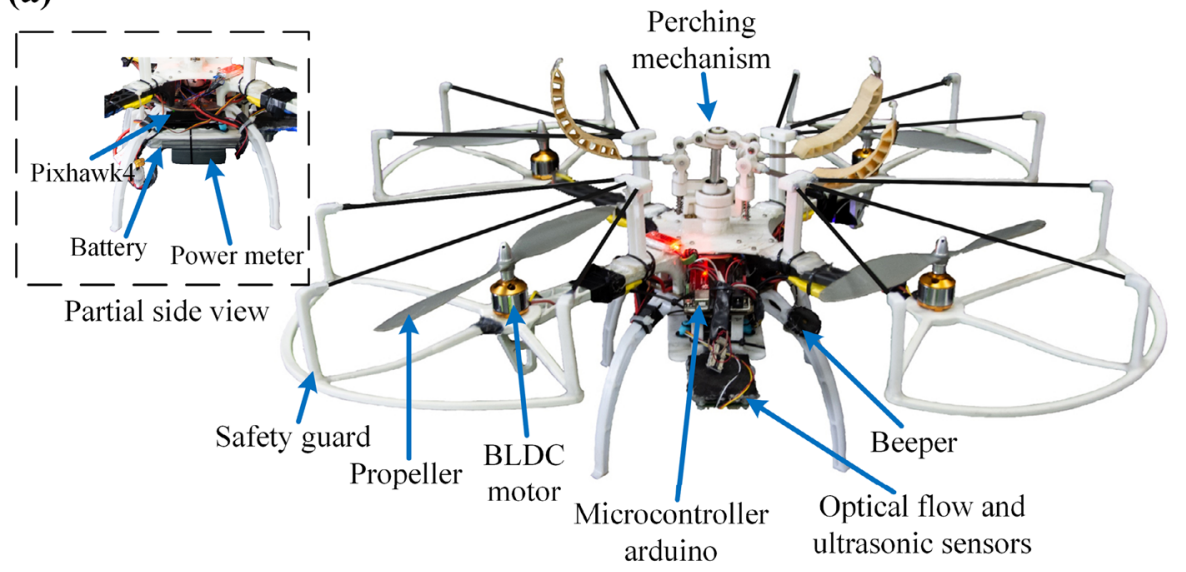

(b)

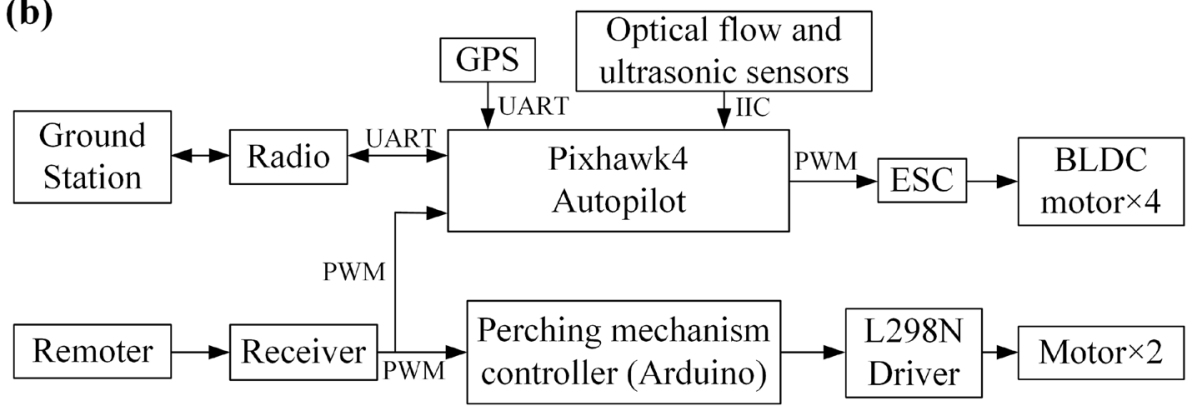




\section{Experiments and Analysis of the Mechanism}

In order to accurately demonstrate the loading capacity of the perching mechanism and its adaptability towards different objects, a test platform (Fig. 5a) is designed and constructed, on which the perching mechanism is fixed on the frame, with its upper part grasping test objects, and its lower part connecting to a tension sensor. During the experiments, the loading value applied to the mechanism is transmitted to a computer by the tension sensor through a digital transmitter and a RS485-USB module and recorded by the computer in real-time. Meanwhile, the other end of the tension sensor is connected to a driving rope, which is connected to a reel through a fixed pulley, with the reel being driven by a DJI M3508 brushless DC motor and the motor speed being controlled by a STM32 microcontroller through an Electronic Speed Controller (ESC). Since the weight of a small UAV is not exceeding $2 \mathrm{~kg}$ and a safety factor of 2 is selected, then $4 \mathrm{~kg}$ is set as the loading value in the test. In order to prevent the perching mechanism from being damaged due to overloading, which would affect subsequent experiments, a PID closed-loop control strategy is adopted accordingly. The test platform and control strategy are shown in Fig. 5.

Three independent experiments are repeated on each of the four types of test targets: cylinder, cuboid, sphere, and cable. For objects with longer lengths in a certain direction, type I configuration, which can be better adapted to the objects' shape, is used. While for objects like spheres, type II configuration is adopted. The process of the performance test is shown in Fig. 6.

Through the experimental results, it can be concluded that the perching mechanism can be adapted to a variety of test objects. Under loading, the toe bones are significantly stretched; the toe pads are strongly compressed and deformed. Thus, the toes are equipped with excellent passive deformability. For objects with a large cross-sectional area, the toe bones and toe pads play a major role in greatly improving the perching mechanism's adaptability with their elasticity and porous structure. For objects with small cross-sectional areas or low hardness, the perching is mainly realized by the claws hooking on suitable surfaces as the effective contacting surface between the objects and toe pads is comparatively small, resulting in large relative sliding. With the relative sliding, the toe pads can be fitted more closely to the surfaces of the perched objects, and the claws can embed into or hook on more suitable surfaces to achieve more stable perching. Additionally, the mechanism can be perched on more types of objects steadily with its configuration-change ability, because of which its adaptability is greatly improved. In addition to those features, the loading value obtained by the tension sensor is plotted into curves, as shown in Fig. 7.

It can be seen that the loading value for various types of test objects can be reached to $4 \mathrm{~kg}$. The mechanism's weight is $258 \mathrm{~g}$, which is only about $1 / 15$ of the set loading value. Thus, the loading capacity of the perching mechanism can fully meet the actual requirements. The time needed to reach the set loading value is the shortest $(2.3 \mathrm{~s})$ when it's perching on the cylindrical object I-a (Fig. 7a) and the longest (4.85 s) on the cable IV-a (Fig. 7d). The reason is that there is a larger contacting area between the mechanism and the cylindrical object, which leads to a more even loading and smaller deformation on the toes. While for the cable, more displacement and deformation are needed by the toes, resulting in a longer loading time. The loading time for other test objects is between that of the cylindrical and the cable.

Meanwhile, it can be observed that objects with higher hardness (Fig. 7c), larger contacting areas (Fig. 7b), and lower

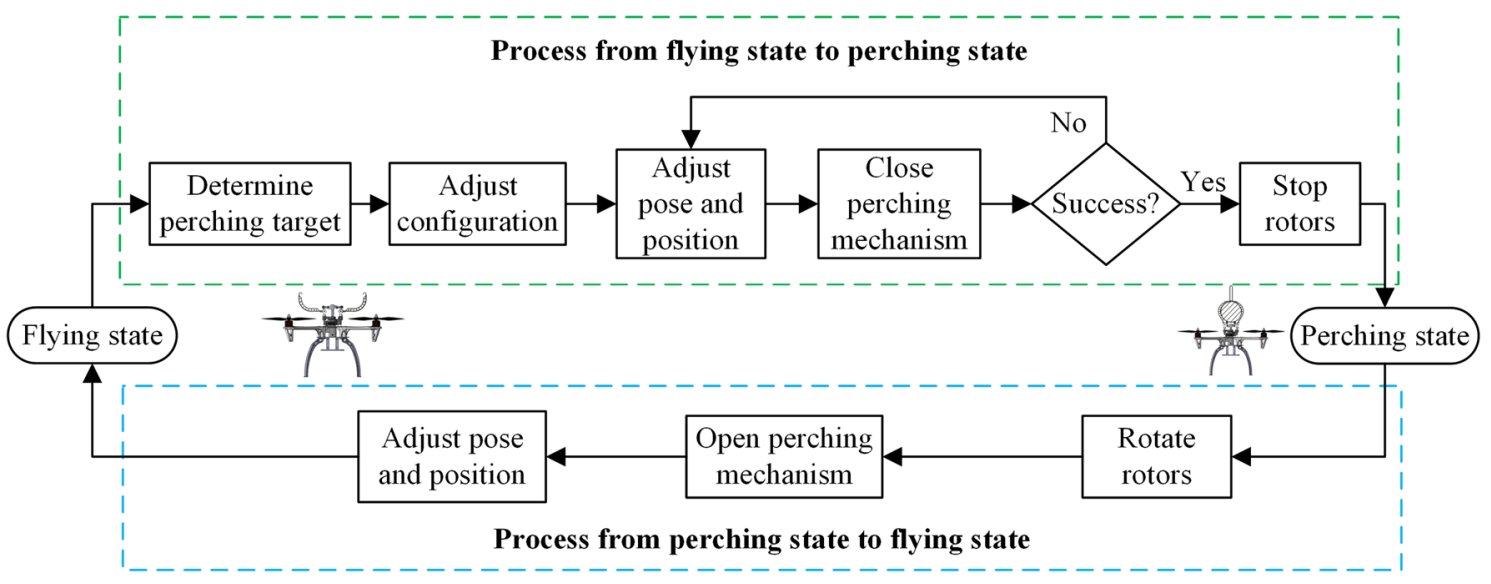

Fig. 9 State-switching strategy. There are two states of the robot, the flying state and the perching state, and the state-switching process is mainly realized by the operator at present. Hopefully, we are investigating a more autonomous way to achieve those processes 


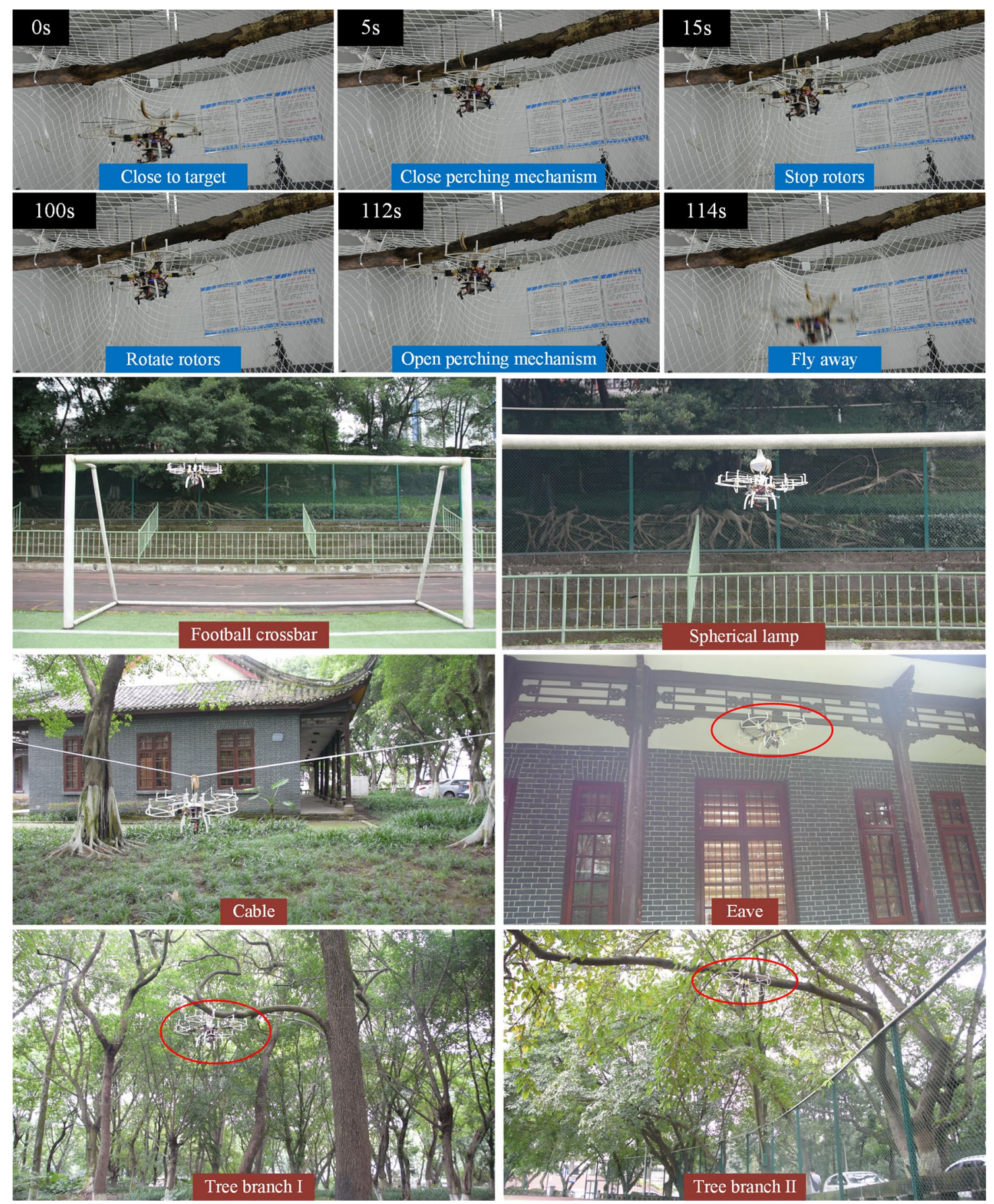

Fig. 10 Flight perching experiments. The first six pictures show the perching processes in a laboratory environment, and the last six pictures show that the system can successfully perch on different targets in outdoor environments

elasticity (Fig. 7d) are equipped with better perching performance (shorter loading time, smoother loading curve, and narrower loading error zone).
In conclusion, the perching mechanism can be well adapted to various objects and easily meet the predetermined loading requirements. 
Table 1 System's specifications

\begin{tabular}{ll}
\hline Name & Parameter \\
\hline Size $(\mathrm{mm})$ & $500(\mathrm{~L}) \times 500(\mathrm{~W})$ (including safety guards) \\
Height $(\mathrm{mm})$ & 510 (including the perching mechanism) \\
Mass $(\mathrm{kg})$ & 1.95 (including all components) \\
Wheelbase $(\mathrm{mm})$ & 450 \\
Flight controller & Pixhawk4 \\
Perching mechanism & Arduino Uno \\
$\quad$ controller & \\
Power supply & Li-Po battery DC $(11.1 \mathrm{~V}, 4000 \mathrm{mAh})$ \\
\hline
\end{tabular}

\section{System Integration and Experiments}

In current aircraft platforms, the multi-rotor UAV is easily controlled with a simple structure. It can take off and land vertically, hover at fixed points. With those advantages, it has been widely used in many fields. Thus, a quadcopter with a DJI F450 airframe is selected as the aerial platform in our research. However, its upper board is redesigned to install the perching mechanism. In addition, Pixhawk4, as an excellent open-source flight controller, is selected as the main controller of the UAV. And it is equipped with optical flow and ultrasonic sensors to realize the function of hovering at fixed points, through which the fluctuation of the aircraft can be greatly reduced, and the success rate of perching can be highly improved. Meanwhile, an Arduino microcontroller is used to control the perching mechanism, and a ZFT8 high-precision curve power meter is adopted to record its power consumption during flight. With the safety guards, the propellers can be partly prevented from colliding with surrounding objects (Fig. 8). System's specifications are listed in Table 1.

To test the stability and reliability of the system, a series of dynamic perching experiments are carried out on different objects, such as tree branches, cables, eaves, and spherical lamps. The experimental process and state-switching strategy are shown in Fig. 9.

As observed, the UAV can be successfully perched on different targets with the perching mechanism (Fig. 10). It can alternate between flying state and perching state accurately and quickly. Its success rate of perching can reach more than $90 \%$ on targets like crossbars, tree branches, and cables, while comparatively lower on spherical objects because more accurate positioning is needed. Overall, the success rate of perching is quite high on all the aforementioned objects.

To further compare the UAV's endurance capabilities with traditional UAV systems in these scenarios, the energy consumption of the perching system during the whole process is recorded by the power meter. The three experimental processes shown in Table 2 are designed to analyze the power consumption better, and three independent experiments are carried out in each experimental process.

The power curve of process I is shown in Fig. 11a, which demonstrates that the experimental results obtained from the three independent experiments are almost the same. Without the perching mechanism, the aircraft's power consumption is stable at around $325 \mathrm{~W}$ during the whole flight. With the battery of $4000 \mathrm{mAh}$ and $11.1 \mathrm{~V}$, the continuous flight time of the UAV is expected as about $8.2 \mathrm{~min}$.

For process II, the power consumption (Fig. 11b) is slightly higher (about $350 \mathrm{~W}$ ) comparing to that in process I. It can be concluded that the power consumption produced by the perching mechanism accounts for about $7.1 \%$ of that of the whole system, and the flight time is estimated as about $7.6 \mathrm{~min}$. However, the small increase in power consumption due to the perching mechanism is acceptable. In addition, the total power consumption is slightly increased due to the heating of the motors and other electronic components with the increase of flying time.

For process III (Fig. 11c), the system's power consumption is almost the same as that in process II during taking off and flight. However, it drops rapidly to about $5 \mathrm{~W}$ when it is perched on targets and the rotors are turned off, which is only $1.4 \%$ of that when hovering. If the UAV is immediately switched to the perching state after a short distance flight, its continuous perching time can reach more than $8 \mathrm{~h}$. Additionally, It is worth noting that the power consumption increases significantly for a short time during the state-switching process, which is caused by adjusting the relative position between the perching mechanism and the targets to achieve successful perching. Meanwhile, certain turbulences will

Table 2 Design of experimental processes

Process Contents

I Without the perching mechanism, the UAV takes off to a height of about $2 \mathrm{~m}$ above the ground, then hovers at a fixed point for a specified time, and finally lands and turns off rotors

II With the perching mechanism, the UAV takes off to a height of about $2 \mathrm{~m}$ above the ground, then hovers at a fixed point for a specified time, and finally lands and turns off rotors

III With the perching mechanism, the UAV takes off to a height of about $2 \mathrm{~m}$ above the ground, then perches on a target and turns off rotors for a specified time, finally flies away, lands, and turns off rotors 

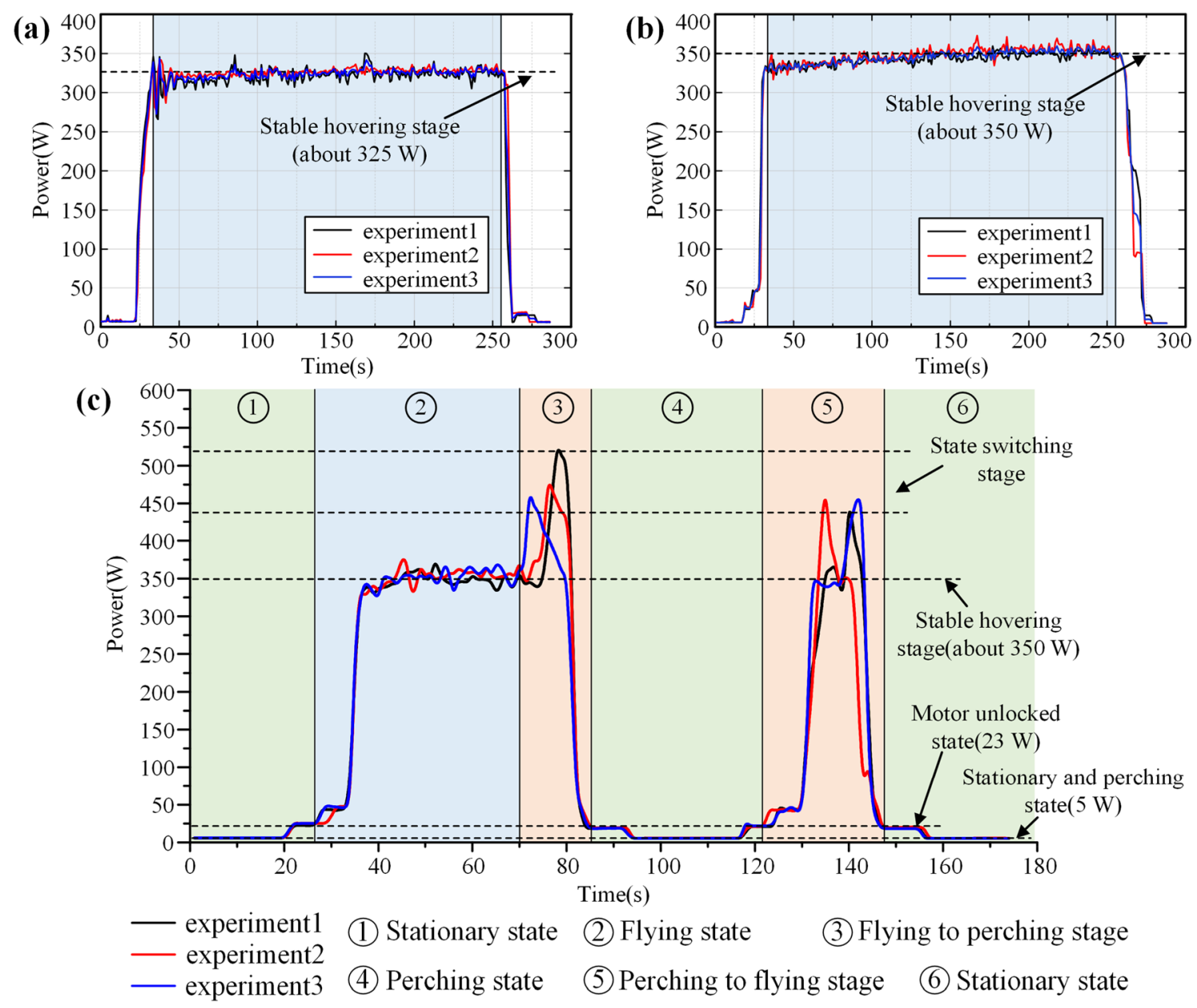

Fig. 11 Power consumption curves. a Process I, without the perching mechanism, the power consumption stable at about $325 \mathrm{w}$ during the hovering stage. b Process II with the perching mechanism, the power consumption increase slightly and stable at about $350 \mathrm{w}$ dur-

be caused by the interaction between the target and the airflow driven by propellers.

In conclusion, the designed system's power consumption during flight is almost the same as that of the UAV without the perching mechanism. However, the UAV with the perching mechanism can be perched on tree branches, eaves, cables, and other elevated objects when performing tasks like fixed-point surveillance, through which its endurance can be greatly increased and concealment can be highly improved.

\section{Conclusions and Future Work}

To improve the endurance, concealment, and adaptability of the existing flying perching robots, a deformable bird-inspired UAV perching mechanism is designed and ing the hovering stage. c Process III with the perching mechanism, the system can perch on objects in outdoor environments. Its power consumption drops drastically to about $5 \mathrm{~W}$, so its endurance time can be greatly improved

presented in this paper. Afterward, experiments are carried out to analyze its adaptability and loading capacity when perching on various objects with different shapes and sizes. By integrating it with a quadcopter, multi-scenario dynamic flying perching experiments are conducted and power consumption is recorded accordingly. Results show that it has strong adaptability and high loading capacity on various types of objects, and the system's power consumption is extremely low when perching on targets. Therefore, it is verified as feasible in the application.

In future research, the robot vision and onboard computer will be considered to realize autonomous perching towards typical targets. Simultaneously, researches about how to make the robot perch on inclined and vertical objects will also be carried out to further improve its adaptability. 
Supplementary Information The online version contains supplementary material available at https://doi.org/10.1007/s42235-021-00098-5.

Acknowledgements This work was financially supported by the National Key R \& D Program of China [Grant No. 2020YFB1313000], National Natural Science Foundation of China [Grant No. 51975070, 62003060, 62073211].

\section{Declarations}

Conflict of Interest The authors declare no competing or financial interests.

Open Access This article is licensed under a Creative Commons Attribution 4.0 International License, which permits use, sharing, adaptation, distribution and reproduction in any medium or format, as long as you give appropriate credit to the original author(s) and the source, provide a link to the Creative Commons licence, and indicate if changes were made. The images or other third party material in this article are included in the article's Creative Commons licence, unless indicated otherwise in a credit line to the material. If material is not included in the article's Creative Commons licence and your intended use is not permitted by statutory regulation or exceeds the permitted use, you will need to obtain permission directly from the copyright holder. To view a copy of this licence, visit http://creativecommons.org/licenses/by/4.0/.

\section{References}

1. Alzahrani, B., Oubbati, O. S., Barnawi, A., Atiquzzaman, M., \& Alghazzawi, D. (2020). UAV assistance paradigm: state-of-the-art in applications and challenges. Journal of Network and Computer Applications, 166, 102706.

2. Shakhatreh, H., Sawalmeh, A. H., Al-Fuqaha, A., Dou, Z. C., Almaita, E., Khalil, I., Othman, N. S., Khreishah, A., \& Guizani, M. (2019). Unmanned aerial vehicles (UAVs): a survey on civil applications and key research challenges. IEEE Access, 7, 48572-48634.

3. Outay, F., Mengash, H. A., \& Adnan, M. (2020). Applications of unmanned aerial vehicle (UAV) in road safety, traffic and highway infrastructure management: Recent advances and challenges. Transportation Research Part A Policy and Practice, 141, 116-129.

4. Liu, Y., Chen, H.P., Tang, Z.M., Sun, G.X. (2012). A bat-like switched flying and adhesive robot. 2012 IEEE International Conference on Cyber Technology in Automation, Control, and Intelligent Systems (CYBER) (pp. 92-97), Bangkok, Thailand.

5. Wopereis, H.W., Molen, T.D., Post, T.H., Stramigioli, S., Fumagalli, M. (2016). Mechanism for perching on smooth surfaces using aerial impacts. 2016 IEEE International Symposium on Safety, Security, and Rescue Robotics (pp. 154-159), Lausanne, Switzerland.

6. Huang, C. W., Liu, Y., \& Ye, X. (2019). Design, simulation and experimental study of a force observer for a flying-perching quadrotor. Robotics and Autonomous Systems, 120, 103237.

7. Kalantari, A., Mahajan, K., Ruffatto, D., Spenko, M. (2015). Autonomous perching and take-off on vertical walls for a quadrotor micro air vehicle. 2015 IEEE International Conference on Robotics and Automation (ICRA) (pp. 4669-4674), Seattle, American.

8. Thomas, J., Pope, M., Loianno, G., Hawkes, E. W., Estrada, M. A., Jiang, H., Cutkosky, M. R., \& Kumar, V. (2016). Aggressive flight with quadrotors for perching on inclined surfaces. Journal of Mechanisms and Robotics, 8, 051007.
9. He, Q. S., Xu, X. R., Yu, Z. W., Huo, K., Wang, Z. Y., Chen, N., Sun, X. A., Yin, G., Du, P. L., Li, Y., \& Dai, Z. D. (2020). Optimized bio-inspired micro-pillar dry adhesive and its application for an unmanned aerial vehicle adhering on and detaching from a ceiling. Journal of Bionic Engineering, 17, 45-54.

10. Roberts, J.F., Zufferey, J.C., Floreano, D. (2008). Energy management for indoor hovering robots. 2008 IEEE/RSJ International Conference on Intelligent Robots and Systems (pp. 1242-1247), Nice, France

11. Garcia-Rubiales, F.J., Ramon-Soria, P., Arrue, B.C., Ollero, A. (2019). Magnetic detaching system for modular UAVs with perching capabilities in industrial environments. The 2019 International Workshop on Research, Education and Development of Unmanned Aerial Systems (pp.172-176), Cranfield, England.

12. Graule, M. A., Chirarattananon, P., Fuller, S. B., Jafferis, N. T., Ma, K. Y., Spenko, M., Kornbluh, R., \& Wood, R. J. (2016). Perching and takeoff of a robotic insect on overhangs using switchable electrostatic adhesion. Science, 352, 978-982.

13. Pope, M. T., Kimes, C. W., Jiang, H., Hawkes, E. W., Estrada, M. A., Kerst, C. F., Roderick, W. R. T., Han, A. K., Christensen, D. L., \& Cutkosky, M. R. (2017). A multimodal robot for perching and climbing on vertical outdoor surfaces. IEEE Transactions on Robotics, 33, 38-48.

14. Hang, K. Y., Lyu, X. M., Song, H. R., Stork, J. A., Dollar, A. M., Kragic, D., \& Zhang, F. (2019). Perching and resting-A paradigm for UAV maneuvering with modularized landing gears. Science Robotics., 4, eaau6637.

15. Doyle, C. E., Bird, J. J., Isom, T. A., Kallman, J. C., Bareiss, D. F., Dunlop, D. J., King, R. J., Abbott, J. J., \& Minor, M. A. (2013). An avian-inspired passive mechanism for quadrotor perching. IEEE/ASME Transactions on Mechatronics, 18, 506-517.

16. Xie, P., Ma, O., Zhao, Z., Zhang, L. (2015). A bio-inspired UAV leg-foot mechanism for landing, grasping and perching tasks. AIAA Atmospheric Flight Mechanics Conference (p. 1689). Kissimmee, American.

17. Nadan, P. M., Anthony, T. M., Michael, D. M., Pflueger, J. B., Sethi, M. S., Shimazu, K. N., Tieu, M., \& Lee, C. L. (2019). A bird-inspired perching landing gear system. Journal of Mechanisms and Robotics., 11, 061002.

18. Roderick, W. R. T., Chin, D. D., Cutkosky, M. R., \& Lentink, D. (2019). Birds land reliably on complex surfaces by adapting their foot-surface interactions upon contact. eLife, 8 , e46415.

19. Chi, W. C., Low, K. H., Hoon, K. H., Tang, J., \& Go, T. H. (2012). A bio-inspired adaptive perching mechanism for unmanned aerial vehicles. Journal of Robotics and Mechatronics, 24, 642-648.

20. Xie, C., Wu, X., \& Wang, X. J. (2019). A three-row opposed gripping mechanism with bioinspired spiny toes for wall-climbing robots. Journal of Bionic Engineering, 16, 994-1006.

21. Beckers, J., Verstraten, T., Verrelst, B., Contino, F., \& Mierlo, J. V. (2021). Analysis of the dynamics of a slider-crank mechanism locally actuated with an act-and-wait controller. Mechanism and Machine Theory, 159, 104253.

22. Baird C, Nokleby S. (2020). Optimization and design of a gripper mechanism for autonomous unmanned aerial vehicle perching. USCToMM Symposium on Mechanical Systems and Robotics (pp. 160-171), Rapid City, American.

23. Chi, W.C., Low, K.H., Hoon, K.H., Tang, J. (2014). An optimized perching mechanism for autonomous perching with a quadrotor. IEEE International Conference on Robotics and Automation (ICRA) (pp. 3109-3115), Hong Kong, China.

24. Stavenuiter, R. A. J., Birglen, L., \& Herder, J. L. (2017). A planar underactuated grasper with adjustable compliance. Mechanism and Machine Theory, 112, 295-306.

Publisher's Note Springer Nature remains neutral with regard to jurisdictional claims in published maps and institutional affiliations. 